Editorial

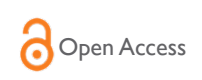

\title{
New trends in small animal surgery
}

\section{Editorial}

Dear Colleagues, It is readily accepted in our era that small animal surgery moves forward very fast taking advantage of the developments and achievements in human surgery. Around the world almost all available for humans surgical devices and instruments and even surgical techniques are also available for use and many of those are already implemented in small animal surgery. Advances in diagnostic imaging (i.e. computed radiography, ultrasonography, computed tomography and magnetic resonance imaging) along with the use of endoscopy provide veterinary surgeons with advanced diagnostic capabilities.

Operating rooms also have more similarities than ever before. Almost all sterilization methods of surgical instruments and equipments including steam, chemicals, plasma and ionizing radiation are available. Advances in anesthesiology, pain management and intensive care make general anaesthesia safer than ever. Aseptic surgical techniques are implemented widely whilst antimicrobial agents are used very wisely in order to prevent overuse and misuse and avoid creating antimicrobial resistance and at the same time cautiously in order to reduce surgical site infections. Electrocoagulation and various hemostatic agents like gelatin sponges are also widely used for hemostasis. Surgical staples have partially replaced sutures thus resulting in reduced operative time.

Endosurgery is also introduced in small animal practices and all of its advantages are very clear and well documented along with its main disadvantage which is the cost. Surgical trauma is minimal, it is less painful, postoperative hospitalization time is reduced and recovery is faster thus contributing in much better rehabilitation.

Cardiovascular operations, tracheal and urethral stenting, advances in surgical oncology, total hip replacement, developments in neurosurgery and kidney transplantation in cats are among the
Volume 4 Issue 2 - 2016

Stefanos Kladakis

Department of Military Working Dogs, Veterinary Clinic, Greece

Correspondence: Stefanos Kladakis, C' Army Veterinary Hospital, Department of Military Working Dogs, Veterinary Clinic, Thermi, 5700I,Thessaloniki, Greece, Tel +30 23I 046 2425, Email kladakis@gmail.com

Received: November 24, 2016 | Published: November 25, 2016

new modalities we can use to our small animal patients in order to provide them advanced veterinary care and improve their quality of life and also increase their life expectancy. Every day more veterinary surgeons recognise the benefits of these modalities and are seeking to be trained and gain expertise and even more veterinary hospitals around the globe acquire cutting edge equipment to fulfill what their small animal patients need the most nowadays and that is advanced options for veterinary care.

\section{Acknowledgements}

None.

\section{Conflict of interest}

The author of this article has no financial or personal relationship with other people or organizations that could inappropriately influence or bias the content of this paper. 